\title{
Minimally Invasive Approach to Urachal Remnant in Obese Patient
}

\author{
Aleksandr A. Reznichenko
}

\begin{abstract}
Urachal anomalies are rare. Most commonly they are clinically present in childhood. Rarely could they be seen in adult patients. These anomalies can cause recurrent infection. The standard of care for this disease is complete resection of all anomalous tissue including a bladder cuff to avoid recurrence. The traditional surgical approach includes large transverse or midline infraumbilical incision. Several reports have shown that minimally invasive technique could be successful in patients with urachal remnant. We present one patient with morbid obesity and urachal remnant who was successfully treated with complete laparoscopic resection.
\end{abstract}

Keywords: Urachal remnant; Laparoscopy; Morbid obesity

\section{Introduction}

Urachus is fibrous remnant of the allantois, a canal that drains the urinary bladder of the fetus that joins and runs within the umbilical cord [1]. It lies in the space of Retzius, between the transversalis fascia anteriorly and the peritoneum posteriorly. By the fourth or fifth month of gestation, the urinary bladder descends into the pelvis and its apical portion progressively narrows to a small, epithelialized fibromuscular strand, the urachus [2]. Urachal remnants represent a failure in the obliteration of the allantois at birth that connects the bladder to the umbilicus [3]. Urachal anomalies are found in 1.6\% of children less than 15 years of age and in $0.063 \%$ of adults [4]. There may be a slight male gender bias in the occurrence of urachal anomalies, with a ratio of $1.2: 1$ to $2: 1$ in pediatric and adult patients, respectively, but rates vary among series given the low numbers reported [5].

Urachal anomalies can be described based on the extent of urachal patency as a patent urachus (entire tract patent), urachal

Manuscript accepted for publication November 13, 2014

Department of Surgery, Adventist Health Medical Center, 115 Mall Dr, Hanford, CA 93230, USA. Email: areznik9@yahoo.com

doi: http://dx.doi.org/10.14740/jes245w cyst/alternating sinus, umbilical-urachus sinus (umbilical side patent), and vesico-urachal diverticulum (bladder side patent) occurring in $50 \%, 30 \%, 15 \%$, and $3-5 \%$ of urachal abnormalities, respectively [6-8]. The persistence of the urachal lumen manifests in several clinical presentations, of which recurrent periumbilical discharge is the most common [9], following by abdominal mass. Very rare other clinical scenarios have been described, including urachal carcinoma $[10,11]$, colo-urachalcutaneous fistula [12] and small bowel obstruction [13]. Diagnosis of the persistent urachal remnant could be challenging and first of all requires a thorough history and physical examination [5]. Various imaging modalities have been used to confirm the diagnosis. They included ultrasonography, computed tomography, voiding cystourethrography, sinography [9] and magnetic resonance imaging $[5,10,14]$. However, none of them are perfect in the identification of urachal remnant. For example, diagnostic accuracy of ultrasound was reported from $61 \%$ to $91 \%$ [9]. Diagnostic laparoscopy could be useful as a diagnostic modality if other tests were inconclusive, and also enable medical management [9].

The traditional approach for removing urachal remnant and for the treatment of complicated urachal cysts has been open surgery $[15,16]$. However, open surgery is associated with increased morbidity, more significant blood loss, later resumption of food intake and longer hospital stay [17]. Initial minimally invasive surgical approaches were described in 1992 and 1993 by Neufang et al [18] and Trondsen et al [19], who performed the first reported laparoscopic excisions of an urachal fistula and sinus, respectively. There were several recent series describing successful laparoscopic procedures for urachal remnant. The most prominent series included Patrzyk et al [20], Siow et al [9] and Araki et al [15] with 21, 14 and eight patients described, respectively. Since first application of minimally invasive technique in 1992, we found 90 cases in the literature of successful laparoscopic resection of urachal remnant in adults [15-30]. Robotic-assisted approach [11] and single incision laparoscopy $[21,22]$ have been reported with equal to conventional laparoscopy success.

General recommendation is wide excision of entire urachal remnant from immediately caudal to the umbilicus down to the bladder dome $[9,15,16,21,23-25]$. Most of the surgeons also agree that excision of the bladder cuff is appropriate when urachal remnant communicates with the bladder [15, 21, 25]. Bladder cuff excision is also necessary if there is suspi- 


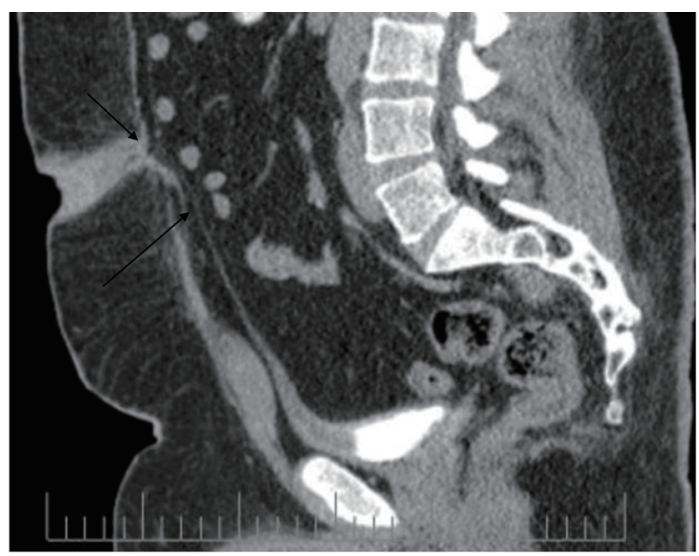

Figure 1. Inflamed umbilicus (short arrow) and urachal remnant (long arrow).

cion of urachal carcinoma [31]. There is no consensus about the resection of the umbilicus. Some advocates resection for resolution of symptoms and superior cosmetic results [15]. Others emphasize importance of meticulous dissection of the urachal remnant together with all inflamed tissues over umbilectomy [9].

We found only one report of laparoscopic management of urachal remnant in morbidly obese patient [26]. Gregory et al reported successful intervention on a 63-year-old female with BMI of 49, who presented with painful swelling in the umbilical region associated with umbilical hernia. Laparoscopic removal of urachal cyst together with hernia repair was performed with excellent outcome.

We present our case of a 30-year-old male with urachal remnant and morbid obesity who was successfully treated with laparoscopic resection.

\section{Case Report}

A 30-year-old male with history of morbid obesity (BMI 43), HTN, DM and laparoscopic cholecystectomy 10 years ago presented with tenderness and foul smelling discharge from his umbilicus for 8 months. He had several courses of ABX with temporally relief. On physical exam, a sinus tract opening was identified at the bottom of his naval with purulent discharge.

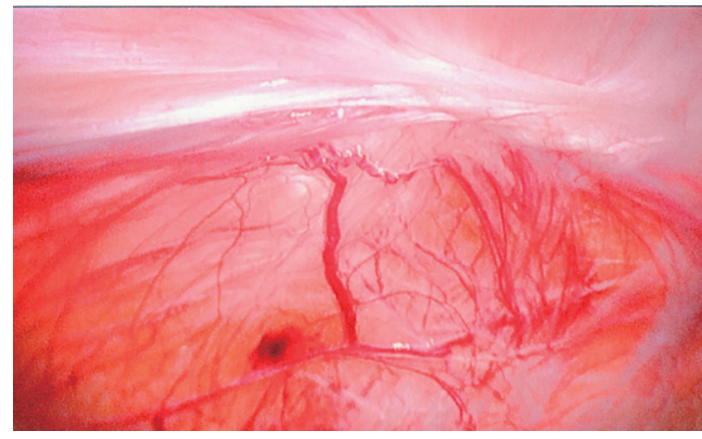

Figure 2. Urachal sinus

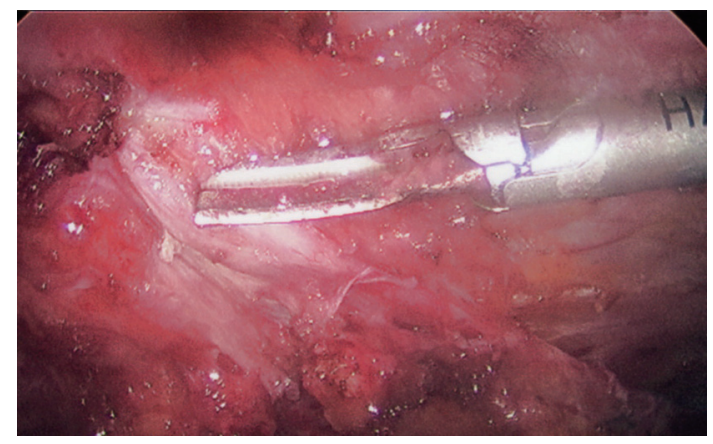

Figure 3. Dissection of urachal sinus.

Sinogramm was inconclusive. Voiding cystogramm was normal. CT showed urachal remnant connecting urinary bladder and umbilicus (Fig. 1). Patient received 5 days PO ABX preoperatively to decrease inflammation in the umbilical skin.

\section{Surgical technique}

After the consent was signed, he was brought to the OR, placed in the supine position, and was intubated by anesthesia. He received IV fluids and IV antibiotics. A Foley catheter and an NG tube were introduced. The patient was prepped and draped in the usual sterile surgical fashion. Umbilical skin had significant chronic and subacute inflammatory changes. Entrance to the intraabdominal cavity through the umbilicus was abandoned. A $15 \mathrm{~mm}$ incision was performed in the left upper quadrant, and the abdomen was entered with Optiview $10 \mathrm{~mm}$ trocar. The abdomen was insufflated. Under direct vision, 5 $\mathrm{mm}$ trocars were placed in the epigastric area and in the right upper quadrant. With all three trocars in place, we proceeded with abdominal exploration. The small bowel was run all the way from the ileocecal valve to the ligament of Treitz, and a diagnosis of a vitellin duct abnormality (Meckel's diverticulum) was ruled out. We identified a sinus tract going from the umbilicus down to the urinary bladder which had chronic inflammatory changes (Fig. 2). Umbilical fascia was involved in the inflammation. Abdomen was desufflated. The umbilical skin was completely excised down to the fascia together with superficial part of the urachal sinus tract. A $10 \mathrm{~mm}$ trocar was introduced to the umbilical fascial defect. We utilized all

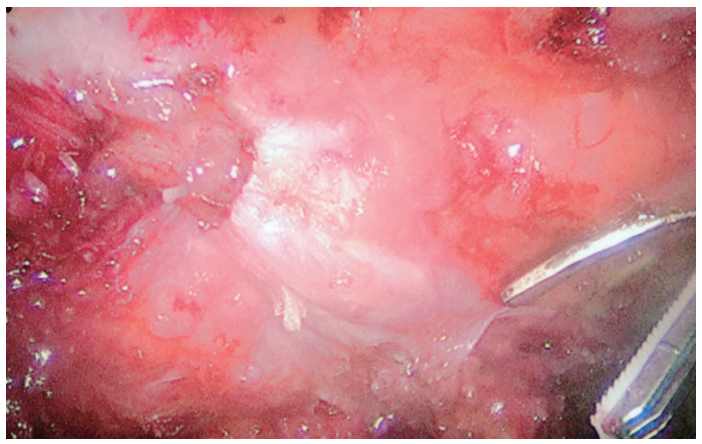

Figure 4. Side deviations of urachal sinus. 


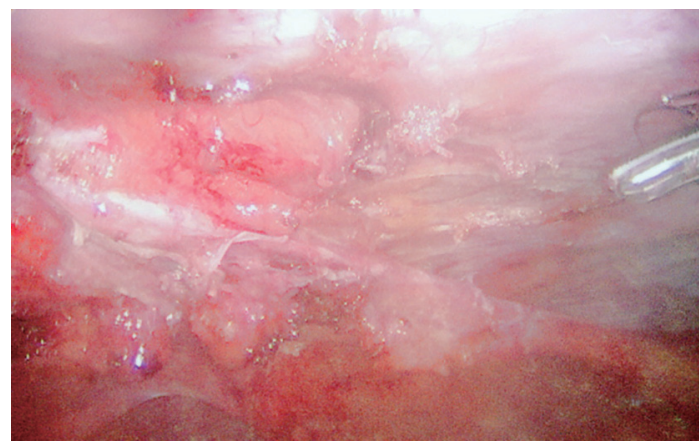

Figure 5. Dissection of side deviations.

four ports. Patient was placed in Trendelenburg position. The urachal sinus tract was dissected from the umbilicus down to the urinary bladder using harmonic scalpel (Fig. 3). All side deviations of the tract on the anterior abdominal wall have been dissected en bloc with major sinus and included into the specimen (Fig. 4, 5). The urinary bladder was filled with 400 $\mathrm{mL}$ of normal saline mixed with $50 \mathrm{~mL}$ of methylene blue. The cuff of the bladder wall was transected with EndoGIA stapler, US Surgical No. 60, blue load, to incorporate entire urachal sinus. Control for hemostasis. Umbilical fascia was closed with several interrupted No. 1 Vicryl sutures using laparoscopic fascial closure system. The fascial defect in the left upper quadrant was closed in the same manner. The umbilicus was reconstructed with interrupted 2-0 vicril sutures approximating dermal layer and deep fascia in four quadrants of the umbilical wound. Skin was closed with running 2-0 Monocryl. The left upper quadrant trocar site wound was approximated with interrupted 2-0 Vicryl suture. The abdomen was desufflated. The NG tube was removed. The Foley catheter remained in place. All incisions except the umbilical incision were closed in the same manner using 4-0 SQ Monocryl. Local anesthesia $30 \mathrm{~mL}$ was used, which was a mixture of 1\% lidocaine and $0.5 \%$ marcaine with epinephrine. The patient was extubated in the OR and transported in stable condition to the recovery room. Pathology showed urachal sinus tract with active and chronic inflammation. Patient made uneventful recovery and had no recurrence 5 years after surgery.

\section{Discussion}

Knowledge of urachal embryology is important to understand clinical significance of the urachal anomalies. Urachus is the embryologic remnant of the allantois and is found among medial umbilical ligaments which themselves represent remnants of fetal umbilical arteries. As fetus grows, the urachus turns into a fibrous cord originating in the apex of the bladder and extending to the umbilicus. The urachus has a length from 3 to $10 \mathrm{~cm}$ and a diameter from 8 to $10 \mathrm{~mm}$. Urachal remnants represent a failure of the obliteration of the allantois. Urachal anomalies are found in $1.6 \%$ of children and $0.063 \%$ of adults. These anomalies could be present as a patent urachus (entire tract patent), urachal cyst/alternating sinus, umbilical-urachus sinus (umbilical side patent), and vesico-urachal diverticulum (bladder side patent)

Clinical presentation of urachal anomalies could be different but the most common is persistent purulent discharge from the naval. Patients usually present with long history of purulent drainage with just temporally relief from ABX treatment. Physician should be aware of atypical presentation of urachal remnant, such as abdominal mass, fistulas, bowel obstruction and malignancy.

Diagnosis of persistent urachal remnant represents a challenge. Physical exam and history remain extremely important. Persistent purulent drainage from the naval together with identification of the opening or sinus tract on the bottom of the naval should always rise suspicious for urachal anomaly. Multiple diagnostic modalities are used to identify urachal remnant. They included voiding cystourethrography, ultrasonography, sinography, computer tomography, magnetic resonance imaging and diagnostic laparoscopy. We agree that laparoscopy could be important not only in establishing diagnosis, but also in management of urachal remnant. This is particularly true in obese patients, in whom visualization of the urachal remnant could be challenging on the imaging studies.

Persistent urachal remnants require surgical treatment. Operation includes wide excision of entire urachal remnant from the naval down to the bladder dome and with bladder cuff if it is involved in the process. Resection of the umbilicus could be necessary but it is controversial. We performed umbilectomy in our patient because of significant changes of the umbilical skin.

Traditionally surgery for urachal anomalies was done utilizing large transverse or midline infraumbilical incision, but it was associated with increased morbidity, more significant blood loss, later resumption of food intake and longer hospital stay. Minimally invasive approach has obvious advantages and has been utilized recently in treatment of urachal anomalies. Since 1992 several series and case reports have been published and included 90 patients with only one report of laparoscopic procedure in morbidly obese patient.

We present a case of urachal remnant in a 30-year-old morbidly obese male patient with BMI of 43. We performed successful elective laparoscopic resection of urachal sinus following preoperative course of PO ABX to decrease inflammation. Principles of laparoscopic technique were universal and included initial three ports with triangulation, following placement of an umbilical port after excision of chronically inflamed umbilical skin together with the superficial end of urachal sinus. We performed umbilectomy because of significant inflammatory changes of the umbilical skin. We emphasize the importance of placement of the initial trocar away from infected naval. In our case urachal sinus had several side deviations which were dissected en bloc with major sinus and included into the specimen. Dissection was done from the umbilicus down to the urinary bladder. Entire urachal sinus was removed after cuff of the bladder transected with stapler.

In conclusion, urachal anomalies in adults are rare and require surgery. Minimally invasive approach allows confirming the diagnosis and performing the complete resection with minimal morbidity, which is particularly true in morbidly obese individuals. 


\section{Acknowledgement}

None.

\section{References}

1. Larsen. Human Embryology. 3rd ed. pp. 258.

2. Moore KL. The urogenital system. In: Moore KL, eds. The developing human. 3rd ed. Philadelphia, Pa: Saunders, 1982; 255-297.

3. Blichert-Toft M, Nielsen OV. Congenital patient urachus and acquired variants. Diagnosis and treatment. Review of the literature and report of five cases. Acta Chir Scand. 1971;137(8):807-814.

4. Ueno T, Hashimoto H, Yokoyama H, Ito M, Kouda K, Kanamaru H. Urachal anomalies: ultrasonography and management. J Pediatr Surg. 2003;38(8):1203-1207.

5. Laboccetta LT, Picard JC. AUA Update Series, Lesson 9. 2012;31. The Urachus: A Review and Update, Department of Urology, Medical University of South Carolina, Columbia, South Carolina, pp. 2.

6. Berman SM, Tolia BM, Laor E, Reid RE, Schweizerhof SP, Freed SZ. Urachal remnants in adults. Urology. 1988;31(1):17-21.

7. Frimberger D, Kropp BP. Bladder anomalies in children. In: Wein AJ, Kavoussi LR, Novick AC, Partin AW, Peters CA, Editors, Campbell-Walsh Urology (9th ed). Philadelphia (PA): Saunders Elsevier. 2007, pp. 3576-3579.

8. Galati V, Donovan B, Ramji F, Campbell J, Kropp BP, Frimberger D. Management of urachal remnants in early childhood. J Urol. 2008;180(4 Suppl):1824-1826; discussion 1827.

9. Siow SL, Mahendran HA, Hardin M. Laparoscopic management of symptomatic urachal remnants in adulthood. Asian J Surg. 2014.

10. Gleason JM, Bowlin PR, Bagli DJ, Lorenzo AJ, Hassouna T, Koyle MA, Farhat WA. A Comprehensive Review of Pediatric Urachal Anomalies and Predictive Analysis for Adult Urachal Adenocarcinoma Development. J Urol. 2014.

11. Madeb R, Knopf JK, Nicholson C, Donahue LA, Adcock B, Dever D, Tan BJ, et al. The use of robotically assisted surgery for treating urachal anomalies. BJU Int. 2006;98(4):838-842.

12. Peters AL, Kruijer MJ, Wiese H, Verbeek PC. A colo-urachal-cutaneous fistula in an 88-year-old male. Int J Surg Case Rep. 2012;3(2):55-58.

13. Vaziri K, Ponsky TA, White JC, Orkin BA. Urachal remnant small bowel obstruction: report of two adult cases. South Med J. 2005;98(8):825-826.

14. Pust A, Ovenbeck R, Erbersdobler A, Dieckmann KP. Laparoscopic management of patent urachus in an adult man. Urol Int. 2007;79(2):184-186.

15. Araki M, Saika T, Araki D, Kobayashi Y, Uehara S, Wa- tanabe T, Yamada K, et al. Laparoscopic management of complicated urachal remnants in adults. World $\mathrm{J}$ Urol. 2012;30(5):647-650.

16. Castillo OA, Vitagliano G, Olivares R, Sanchez-Salas R. Complete excision of urachal cyst by laparoscopic means: a new approach to an uncommon disorder. Arch Esp Urol. 2007;60(5):607-611.

17. Okegawa T, Odagane A, Nutahara K, Higashihara E. Laparoscopic management of urachal remnants in adulthood. Int J Urol. 2006;13(12):1466-1469.

18. Neufang T, Ludtke FE. Laparoscopic excision of an urachal fistula - new therapy for a rare disorder. Min Invasive Ther. 1992;1:245.

19. Trondsen E, Reiertsen O, Rosseland AR. Laparoscopic excision of urachal sinus. Eur J Surg. 1993;159(2):127128.

20. Patrzyk M, Wilhelm L, Ludwig K, Heidecke CD, von Bernstorff W. Improved laparoscopic treatment of symptomatic urachal anomalies. World J Urol. 2013;31(6):14751481.

21. Sato F, Shin T, Yuki H, Liang M, Ando T, Mori K, Nomura $T$, et al. Umbilical laparoendoscopic single-site technique for complete excision of urachal remnant. J Laparoendosc Adv Surg Tech A. 2012;22(9):899-903.

22. Patrzyk M, Glitsch A, Schreiber A, von Bernstorff W, Heidecke CD. Single-incision laparoscopic surgery as an option for the laparoscopic resection of an urachal fistula: first description of the surgical technique. Surg Endosc. 2010;24(9):2339-2342.

23. Permpongkosol S, Bella AJ, Suntisevee S, Leenanupunth C, Stoller ML. Laparoscopic excision of urachal cysts in elderly men and woman following pregnancy. J Med Assoc Thai. 2010;93(1):132-136.

24. Cutting CW, Hindley RG, Poulsen J. Laparoscopic management of complicated urachal remnants. BJU Int. 2005;96(9):1417-1421.

25. Cadeddu JA, Boyle KE, Fabrizio MD, Schulam PG, Kavoussi LR. Laparoscopic management of urachal cysts in adulthood. J Urol. 2000;164(5):1526-1528.

26. Gregory GC, Vijay R, Ligaj M, Shiwani MH. Laparoscopic management of urachal cyst associated with umbilical hernia. Hernia. 2011;15(1):93-95.

27. Jeong HJ, Han DY, Kwon WA. Laparoscopic management of complicated urachal remnants. Chonnam Med J. 2013;49(1):43-47.

28. Jorion JL. Laparoscopic removal of urachal cyst. J Urol. 1994;151(4):1006-1007.

29. Siegel JF, Winfield HN, Valderrama E, Smith AD. Laparoscopic excision of urachal cyst. J Urol. 1994;151(6):16311633.

30. Stone NN, Garden RJ, Weber H. Laparoscopic excision of a urachal cyst. Urology. 1995;45(1):161-164.

31. Sheldon CA, Clayman RV, Gonzalez R, Williams RD, Fraley EE. Malignant urachal lesions. J Urol. 1984;131(1):18. 